\title{
Effect of levodopa on cognitive function in Parkinson's disease with and without dementia and dementia with Lewy bodies
}

\author{
S A Molloy, E N Rowan, J T O'Brien, I G McKeith, K Wesnes, D J Burn
}

See end of article for authors' affiliations

Correspondence to: S A Molloy, Department of Neurology, Newcastle General Hospital,

Westgate Road, Newcastle upon Tyne NE4 6BE, UK; Sophie.molloy@

btopenworld.com

Received 18 May 2006 Accepted 19 August 2006

Published Online First

4 September 2006
Background: Levodopa (L-dopa) is the gold standard treatment for Parkinson's disease, but a lack of clear efficacy combined with a perceived liability to neuropsychiatric side effects has limited L-dopa use in patients with parkinsonism and dementia. Therefore, the effect of L-dopa on the cognitive profile of dementia with Lewy bodies (DLB) and Parkinson's disease with dementia (PDD) is unclear.

Aim: To ascertain the acute and long-term effects of L-dopa on aspects of attention and cognition in patients with DLB and PDD, and to compare these with the effects in Parkinson's disease.

Method: Baseline cognitive and motor function was assessed off L-dopa in patients with Parkinson's disease $(n=22), \operatorname{PDD}(n=27)$ and DLB $(n=11)$ using standard "bedside" measures and a computerised programme detecting reaction times and accuracy. All patients then underwent an acute L-dopa challenge with subsequent subjective and objective analysis of alertness, verbal recall, reaction times and accuracy. The same parameters were measured after 3 months on L-dopa to assess the prolonged effect.

Results: Acute L-dopa challenge considerably improved motor function and subjective alertness in all patients without compromising either reaction times or accuracy, but increased fluctuations were noted in both groups with dementia. Neuropsychiatric scores improved in patients with Parkinson's disease both with and without dementia on L-dopa at 3 months. Although patients with Parkinson's disease also had better mean global cognitive function at this time, mean verbal attention and memory deteriorated, and patients with PDD had slower reaction times in some tests. No patient had a marked deterioration over this time. Patients with DLB did not experience any adverse cognitive or neuropsychiatric effects after 3 months of L-dopa treatment.

Conclusion: The use of L-dopa in patients with parkinsonism with dementia does not adversely affect cognitive function.
$\mathrm{D}$ ementia occurs six times more often in patients with Parkinson's disease than in age-matched controls, ${ }^{1}$ whereas dementia with Lewy bodies (DLB) is recognised as the second most common form of degenerative dementia in an ageing population. ${ }^{2}$ Early cognitive deficits are found in many patients with Parkinson's disease and are typically disorders of verbal memory, dysexecutive syndromes

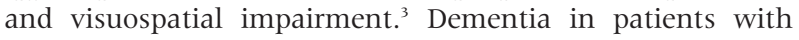
Parkinson's disease characteristically features prominent fluctuating attention and an overall clinical cognitive and neuropsychiatric profile similar to that seen in patients with DLB. ${ }^{4}$ Visual hallucinations are common to both conditions and may reflect drug usage. ${ }^{5}$ Parkinson's disease with dementia (PDD) and DLB are currently differentiated by the temporal evolution of motor and cognitive impairment, with an arbitrary cut-off of 12 months used to differentiate the two dementias. ${ }^{6}$ Distinguishing therapeutic features include neuroleptic sensitivity in patients with DLB, whereas early-diminished verbal fluency and L-dopa-induced confusion may be predictive of incident dementia in patients with Parkinson's disease. ${ }^{7}$ L-dopa improves extrapyramidal signs in patients with Parkinson's disease, PDD and to a variable extent in those with DLB, but may have both beneficial and deleterious effects on cognitive performance and attention in patients with Parkinson's disease. ${ }^{8}$ The influence of this drug on cognition in patients with PDD and DLB has not, to our knowledge, been previously compared, but, notably, the apparent adverse influence of L-dopa on cognitive and behavioural features has led to drug withdrawal in patients with DLB. ${ }^{9}$ Therefore, the treatment of parkinsonism in dementia may be suboptimal owing to the concerns of worsening cognitive and behavioural function.
Many neurobehavioural features in patients with both DLB and PDD reflect underlying cholinergic dysfunction and are responsive to treatment with cholinesterase inhibitors (ChEIs). Attention, apathy, excessive somnolence and hallucinations are most likely to benefit patients with $\mathrm{DLB}^{10}$ whereas in patients with PDD, both cognitive and neuropsychiatric function may also improve with this treatment. ${ }^{11}$ Dopaminergic treatment can potentially exacerbate psychosis, and pharmacokinetic studies have reported an increased risk of adverse events with the concomitant use of L-dopa and ChEIs in patients with Parkinson's disease. ${ }^{12}$

We aimed to clarify the acute and prolonged effects of Ldopa on the cognitive, attentional and neurobehavioural profiles of patients with Parkinson's disease and patients with PDD and DLB on ChEI treatment.

\section{PATIENTS AND METHODS}

All patients were recruited from hospital and community populations under the care of neurologists, psychiatrists and geriatricians. The Newcastle and North Tyneside ethics committee approved the study. All participants gave informed consent to be included; in the case of significant

Abbreviations: ChEl, cholinesterase inhibitors; COG RT, cognition reaction time; CRT, choice reaction time; CRT ACC, CRT response accuracy; CRTSD, CRT standard deviation; CVARCRT, coefficient of variation in choice reaction time; DLB, dementia with Lewy bodies; DPIC RT, delayed picture recognition reaction time; DVIG RT, digit vigilance reaction time; L-dopa, levodopa; MMSE, Mini-Mental State

Examination; NPI, Neuropsychiatric Inventory; NWM RT, numeric working memory reaction time; PDD, Parkinson's disease with dementia; RDS, reverse digit span; SRT, simple reaction time; UPDRS, Unified Parkinson's Disease Rating Scale; VAS, Visual Analogue Scale 
dementia, assent was obtained from the main carer of the patient. Diagnosis was made by agreement between experienced clinicians using the consensus criteria for DLB $^{6}$ and the UK Parkinson's Disease Society Brain Bank criteria for Parkinson's disease. ${ }^{13}$ Patients with PDD conformed to the criteria for Parkinson's disease, but also fulfilled the Diagnostic statistical manual for mental disorders-fourth edition (DSM IV) criteria for dementia ${ }^{14}$ and clinical diagnostic criteria for probable DLB-namely, fluctuating cognition and visual hallucinations, developing more than 12 months after the onset of motor symptoms. ${ }^{6}$ In total, 31 patients with Parkinson's disease, 33 with PDD and 27 with DLB were screened for this study. Of these, 22 patients with Parkinson's disease, 27 with PDD and 11 with DLB completed the acute Ldopa challenge and 3-month follow-up period. Reasons for not participating were lack of regular L-dopa treatment (DLB, $\mathrm{n}=2$ ), side effects of L-dopa (DLB, $\mathrm{n}=4$; two gastrointestinal and two neuropsychiatric), and inability to complete the assessment programme owing to cognitive frailties (DLB, $n=7)$, comorbidities or mortality (DLB, $n=3$ ). Only L-dopa (co-careldopa/co-beneldopa) treatment was allotted for parkinsonism, and $100 \%$ of patients with PDD were on regular treatment for at least 3 years compared with $91 \%$ of patients with DLB and 36\% with Parkinson's disease who were L-dopa naive at study entry. Most patients with dementia had undergone a trial of ChEI treatment at one stage, and during this study $91 \%$ of patients with DLB and $85 \%$ of those with PDD were on a stable dose of one of these agents (donepezil or rivastigmine). Patients with L-dopa-induced dyskinesias were included $(\mathrm{n}=10,9$ patients with PDD and 1 with Parkinson's disease), and all patients were encouraged to use their preferred hand for tasks.

Concomitant depression was diagnosed in two patients with PDD, using the DSM IV criteria. ${ }^{14}$ Although premorbid level of education was not recorded, a Mini-Mental State Examination (MMSE) at study entry provided a global measure of cognition. ${ }^{15}$ Information on neuropsychiatric and neurobehavioural clinical features was obtained using the Neuropsychiatric Inventory (NPI), ${ }^{16}$ which was completed by the same carer at each visit. Subjective change in alertness was calculated using a derivative of the Bond and Lader Visual Analogue Scale (VAS range 0-100, with higher scores implying greater alertness) ${ }^{17}$ Verbal working memory and attention were tested using a reverse digit span (RDS) sequence assessment (derived from the Weschler Adult Intelligence Scale). ${ }^{18}$

Cognitive function was further assessed using tests from the Cognitive Drug Research computerised assessment system that has previously proved sensitive to deficits in patients with parkinsonism and dementia. ${ }^{41}$ The battery included examination of simple (SRT) and choice (CRT) reaction times, CRT response accuracy (CRT ACC), digit vigilance reaction time (DVIG RT), numeric working memory reaction time (NWM RT) and episodic memory, using delayed picture recognition reaction time (DPIC RT). Fluctuating cognition was assessed using CRT standard deviation (CRTSD), representing withintrial variability as described previously. ${ }^{20}$ This information was supported by measuring the coefficient of variation in choice reaction time (CVARCRT), calculated by dividing CRTSD by CRT, controlling for differences in mean performance in that task. Cognitive reaction time was calculated by subtracting SRT from CRT, and provided a measure of cognitive processing time. ${ }^{4}$

In patients with Parkinson's disease, there are two recognised therapeutic motor responses to L-dopa. The short duration response is primarily seen in drug-naive patients but also in advanced disease, on long-term treatment after each dose. ${ }^{21}$ The main recommendation in assessing the short duration response in treated patients is that a positive response should be defined in advance. ${ }^{22}$ For this study, the goal was set in motor terms, whereby an improvement of $>20 \%$ in the Unified Parkinson's Disease Rating Scale (UPDRS) III $^{23}$ indicated a positive therapeutic response. Patients on L-dopa at study entry underwent a baseline cognitive assessment in the morning, after an overnight fast and before their first daily dose of L-dopa, in a practically defined "off" state. ${ }^{24}$ This method has been used in previous motor studies, but has limitations particularly because of an inability to fully exclude the effects of the long duration response to L-dopa, which can last days to months after discontinuation of treatment. ${ }^{21}$ Patients then received dispersible co-beneldopa in a dose equivalent to their regular morning L-dopa dose. Motor and cognitive changes were monitored over 2 hours before they again started their regular daily L-dopa treatment. Patients who were not already on L-dopa at study entry had an identical baseline assessment before starting regular L-dopa at a dose of co-careldopa 12.5/50 three times daily, which was gradually titrated to the maximum tolerated dose according to dose response or systemic side effects encountered over 3 months. No patient had dose escalation limited because of neuropsychiatric side effects. A fasting assessment with acute L-dopa challenge was carried out after 3 months of regular treatment. Therefore, no patient underwent a de novo acute L-dopa challenge.

The assessment schedule included baseline MMSE, NPI, RDS, VAS and cognitive drug research testing, and concluded with an assessment of motor function, using the UPDRS III in all patients while off L-dopa. An acute L-dopa challenge involved repeated SRT, CRT, RDS, VAS and UPDRS III measures at 60,90 and 120 min after treatment. The effects of treatment were calculated by comparing baseline measures off L-dopa with sequential measures after treatment over $2 \mathrm{~h}$. Long-term influence was determined using a full assessment to detect change in MMSE, NPI, RDS, VAS and cognitive drug research at 3 months while on L-dopa compared with the off treatment measures.

\section{STATISTICAL ANALYSIS}

SPSS for Windows (V.11) was used for data analysis. Distributions of baseline data were examined using histograms and Kolmogorov-Smirnov testing. For continuous normally distributed baseline variables, differences across groups were assessed using analysis of variance with retrospective Games-Howell tests to determine pairwise group differences. For non-parametric baseline data, KruskalWallis testing was used to measure differences across groups. $\chi^{2}$ analysis was used to determine differences in frequency data. To determine any differences in performance between diagnostic groups and any within-subject temporal effect of acute treatment response over the 2-h acute test period, sequential acute responses were compared with those at baseline using a mixed between-within subjects analysis of variance (an extension of a repeated-measures design). Log transformation of SRT, CRT, cognition reaction time (COG RT) and CRTSD scores was carried out before analysis because these results were positively skewed, whereas CRT ACC scores were negatively skewed and were thus subtracted from 101 (ie, a maximum score of $100 \%+1$ ) before being $\log$ transformed. Baseline and 3-month test scores were compared using paired t tests or Wilcoxon's signed rank tests as appropriate within each group.

\section{RESULTS}

Patients with PDD were younger (Games-Howell: $p=0.007$ ) but had a longer disease duration (Mann-Whitney $\mathrm{U}$ test: $\mathrm{z}=-3.281, \mathrm{p}=0.001)$ and more severe parkinsonism (Games-Howell: $p=0.016$ ) than those with Parkinson's disease (table 1). As expected, all baseline cognitive measures were better in patients with Parkinson's disease than either group with dementia. Patients with Parkinson's disease also 
reported feeling more alert than those with PDD (GamesHowell: $p<0.001$ ), but not patients with DLB (GamesHowell: $\mathrm{p}=0.386$ ).

\section{Acute L-dopa challenge}

The mean (SD) dose of L-dopa administered acutely was higher in patients with PDD (137 (55) mg) than for patients with either Parkinson's disease ( $105(21) \mathrm{mg} ; \mathrm{p}=0.007$ ) or with DLB (95 (35) mg; $\mathrm{p}=0.041$ ). The mean acute improvement in UPDRS III scores after L-dopa was $22 \%$ for both patients with Parkinson's disease and PDD, whereas for patients with DLB this was just $14.8 \%$. After acute treatment with L-dopa, there was no significant within-subject effect of treatment on SRT, CRT, CRTSD, CRT ACC or RDS scores over $2 \mathrm{~h}$ (table 2). Patients with Parkinson's disease consistently performed better than either group with dementia in SRT, CRT, CRTSD and RDS $(p \leqslant 0.001)$, whereas patients with PDD and DLB were indistinguishable by these measures. Similarly, although there was no change in COG RT in any group after treatment with Ldopa, patients with Parkinson's disease required less cognitive processing time than those with PDD $(\mathrm{p}<0.001)$. No COG RT differences were detected between either patients with Parkinson's disease and DLB or those with PDD and DLB ( $\mathrm{p}=0.194$ and $\mathrm{l}$, respectively). By contrast, L-dopa seemed to acutely influence CVARCRT, VAS and UPDRS III scores $(\mathrm{p}=0.009,0.005$ and $<0.001$, respectively), representing increased CRT fluctuation (despite controlling for mean score), increased subjective alertness and improved motor function after treatment. Patients with Parkinson's disease scored better than those with PDD in CVARCRT, VAS and UPDRS III $(p<0.001, p=0.002, p=0.035$, respectively $)$ and better than DLB in CVARCRT $(p=0.001)$. Also, patients with PDD and DLB could not be differentiated by effect of L-dopa on these measures (CVARCRT, $\mathrm{p}=1$; VAS, $\mathrm{p}=0.158$; UPDRS III; $\mathrm{p}=0.651)$. An interactive effect between time point and diagnosis was apparent for CRT and CRTSD ( $p=0.006$ and 0.033, respectively) which suggests a higher likelihood of fluctuating attention in patients with dementia.

\section{Chronic L-dopa use}

Cognitive parameters off L-dopa were compared with measures on regular L-dopa at 3 months to determine the effect of prolonged use (table 1). The mean (SD) daily dose of L-dopa was larger in patients with PDD (672 (451) $\mathrm{mg}$ ) than in those with Parkinson's disease (407 (197) mg, $\mathrm{p}=0.02$ ) or patients with DLB (286 (105) mg, p=0.001). No difference between baseline and 3-month MMSE scores was apparent for either group with dementia, but the mean MMSE increased in patients with Parkinson's disease by 1.3 points $(t=-2.339$, $\mathrm{p}=0.029)$. RDS scores deteriorated in patients with Parkinson's disease $(\mathrm{t}=2.183, \mathrm{p}=0.040)$ and DVIG RT scores worsened in patients with PDD $(t=-2.145, p=0.041)$ over this time. NPI scores improved in patients with Parkinson's disease either with $(\mathrm{z}=-2.039, \mathrm{p}=0.041)$ or without $(\mathrm{z}=-2.096, \mathrm{p}=0.036)$ dementia, but patients with PDD showed deterioration in SRT $(z=-2.138, p=0.032)$. Accuracy of CRT response did not change over 3 months in any group. Patients with DLB showed no significant change in any measure over this time period. A sensitivity analysis identified whether any person showed cognitive deterioration after 3 months of L-dopa compared with baseline (data not shown). A decline of $<2$ SDs below the mean was considered significant and showed that no individual, in any patient group, declined consistently across more than one cognitive test.

\section{DISCUSSION}

This study showed that L-dopa does not compromise the cognitive or behavioural profiles of patients with parkinsonism and dementia either acutely or over 3 months. After

Table 1 Demographics and baseline and 3-month cognitive data

\begin{tabular}{|c|c|c|c|c|}
\hline & $P D, n=22$ & PDD, $n=27$ & DLB, $n=11$ & $\begin{array}{l}\text { Comparison of baseline characteristics } \\
\text { across groups }\end{array}$ \\
\hline Age (years) & $78.1(6.4)$ & $72.6(5.2)$ & $76.4(6.8)$ & $\mathrm{F}_{2,57}=5.282, \mathrm{p}=0.008^{*}$ \\
\hline Sex, M:F & $17: 5$ & $19: 8$ & $7: 4$ & $\chi^{2}=0.712, p=0.700 \dagger$ \\
\hline Disease duration (years) & $5.0(5.1)$ & $9.4(6.0)$ & $2.8(1.7)$ & $\chi^{2}=19.339, p<0.001 \neq$ \\
\hline No taking levodopa at baseline & 14 & 27 & 1 & $\chi^{2}=31.429, p<0.001 \dagger$ \\
\hline No taking ChEls at baseline & 0 & 23 & 10 & $\chi^{2}=42.560, p<0.001 \dagger$ \\
\hline UPDRS III at baseline & $32.6(9.5)$ & $42.0(13.2)$ & $34.3(12.7)$ & $\mathrm{F}_{2,57}=4.185, \mathrm{p}=0.020^{*}$ \\
\hline Baseline SRT (ms) & $401(94)$ & $696(564)$ & 575 (249) & $\chi^{2}=11.709, p=0.003 \ddagger$ \\
\hline SRT 3 months (ms) & 411 (134) & $855(671)$ & $540(144)$ & \\
\hline Baseline CRT (ms) & $582(108)$ & $1209(906)$ & $1055(560)$ & $\chi^{2}=21.890, p<0.001 \ddagger$ \\
\hline CRT 3 months (ms) & $579(128)$ & $1245(639)$ & $1286(1009)$ & \\
\hline Baseline CRT ACC (\%) & $96.1(6.5)$ & $85.8(14.5)$ & $85.1(13.2)$ & $\chi^{2}=13.306, p=0.001 \ddagger$ \\
\hline CRT ACC 3 months (\%) & $96.1(4.3)$ & $88.1(13.2)$ & $81.5(18.8)$ & \\
\hline Baseline NWM RT (ms) & $1268(1091)$ & $2966(2609)$ & $4255(4272)$ & $\chi^{2}=15.203, p<0.001 \neq$ \\
\hline NWM RT 3 months (ms) & $932(258)$ & 3229 (2437) & 4313 (2581) & \\
\hline Baseline DVIG RT (ms) & $516(75)$ & $680(140)$ & $697(124)$ & $\mathrm{F}_{2,57}=14.579, \mathrm{p}<0.001^{*}$ \\
\hline DVIG RT 3 months (ms) & $526(92)$ & $751(205)$ & 647 (91) & \\
\hline Baseline DPIC RT (ms) & $1384(1022)$ & $2723(2371)$ & 3550 (3407) & $\chi^{2}=12.757, p=0.002 \ddagger$ \\
\hline DPIC RT 3 months (ms) & 1123 (308) & 2658 (1995) & 4279 (3584) & \\
\hline Baseline RDS & $6.2(2.0)$ & $3.9(1.8)$ & $3.4(2.3)$ & $\mathrm{F}_{2,57}=11.102, \mathrm{p}<0.001^{*}$ \\
\hline RDS 3 months & $5.4(1.5)$ & $3.7(2.1)$ & $3.4(1.4)$ & \\
\hline Baseline VAS & $66.6(12.6)$ & $46.9(16.0)$ & $58.6(17.4)$ & $F_{2,57}=10.429, p<0.001^{*}$ \\
\hline VAS 3 months & $68.2(16.5)$ & $48.2(16.4)$ & $59.8(14.1)$ & \\
\hline Baseline MMSE & $25.6(2.2)$ & $20.7(4.8)$ & $19.5(3.6)$ & $\mathrm{F}_{2,57}=13.641, \mathrm{p}<0.001^{*}$ \\
\hline MMSE 3 months & $26.9(2.5)$ & $19.7(5.2)$ & $18.6(4.6)$ & \\
\hline Baseline NPI & $5.6(11.0)$ & $14.9(15.1)$ & $11.9(12.1)$ & $\chi^{2}=9.025, p=0.011 \ddagger$ \\
\hline NPI 3 months & $2.5(5.4)$ & $9.6(15.4)$ & $8.4(9.6)$ & \\
\hline \multicolumn{5}{|c|}{$\begin{array}{l}\text { ChEls, cholinesterase inhibitors; CRT, choice reaction time; CRT ACC, CRT response accuracy; DLB, dementia with Lewy bodies; DPIC RT, delayed picture } \\
\text { recognition reaction time; DVIG RT, digit vigilance reaction time; MMSE, Mini-Mental State Examination; NPI, Neuropsychiatric Inventory; NWM RT, numeric } \\
\text { working memory reaction time; PD, Parkinson's disease; PDD, Parkinson's disease with dementia; RDS, reverse digit span; SRT, simple reaction time; UPDRS, } \\
\text { Unified Parkinson's Disease Rating Scale; VAS, Visual Analogue Scale. } \\
\text { Values are mean (SD). } \\
\text { *Analysis of variance results. } \\
\dagger \chi^{2} \text { results. } \\
\ddagger \text { Kruskal-Wallis results. }\end{array}$} \\
\hline
\end{tabular}


Table 2 Acute L-dopa challenge data at each time point (mean (SD))

\begin{tabular}{|c|c|c|c|c|c|c|c|c|}
\hline $\begin{array}{l}\text { Outcome } \\
\text { measure }\end{array}$ & Group & $0 \mathrm{~min}$ & $60 \mathrm{~min}$ & $90 \mathrm{~min}$ & $120 \mathrm{~min}$ & $\begin{array}{l}\text { Within-subject } \\
\text { effects* }^{*}\end{array}$ & $\begin{array}{l}\text { Between-subject } \\
\text { effects } †\end{array}$ & Interaction effectsł \\
\hline \multirow[t]{3}{*}{ SRT } & PDD & 701 (562) & $807(812)$ & $661(390)$ & $580(283)$ & $W L=0.974$ & $F_{2,57}=12.35$ & $W L=0.901$ \\
\hline & DLB & 946 (788) & $1109(1128)$ & $905(453)$ & $835(386)$ & $\begin{array}{l}F_{3,55}=0.492 \\
p=0.689\end{array}$ & $\mathrm{p}<0.001$ & $\begin{array}{l}F_{6,110}=0.982 \\
p=0.441\end{array}$ \\
\hline & PD & 401 (94) & $387(76)$ & $390(84)$ & $409(84)$ & $\mathrm{ES}=0.026$ & $E S=0.302$ & $\mathrm{ES}=0.051$ \\
\hline \multirow[t]{3}{*}{ CRT } & PDD & $1223(902)$ & $1548(1535)$ & 1367 (1377) & $1230(1260)$ & $W L=0.970$ & $F_{2,56}=11.85$ & $W L=0.718$ \\
\hline & DLB & $1308(840)$ & 954 (338) & 1508 (1977) & 1407 (1233) & $\mathrm{F}_{3,54}=0.560$ & $\begin{array}{l}\mathrm{p}<0.001 \\
\mathrm{ES}=0.297\end{array}$ & $\begin{array}{l}F_{6,108}=3.249 \\
p=0.006\end{array}$ \\
\hline & PD & $602(117)$ & $589(112)$ & $558(90)$ & 559 (89) & $\begin{array}{l}p=0.643 \\
E S=0.03\end{array}$ & & $\mathrm{ES}=0.153$ \\
\hline \multirow[t]{3}{*}{ CRTSD } & PDD & $652(884)$ & 1195 (1871) & 917 (1660) & 747 (1355) & $W L=0.887$ & $\begin{array}{l}F_{2,56}=19.274 \\
p<0.001 \\
E S=0.408\end{array}$ & $W L=0.779$ \\
\hline & DLB & $645(621)$ & $507(350)$ & 857 (1762) & $784(961)$ & $F_{3,54}=2.285$ & & $\begin{array}{l}F_{6,108}=2.390 \\
p=0.033\end{array}$ \\
\hline & PD & $128(47)$ & $141(71)$ & $128(71)$ & $141(70)$ & $\begin{array}{l}p=0.089 \\
E S=0.113\end{array}$ & & $\mathrm{ES}=0.117$ \\
\hline \multirow[t]{3}{*}{ CRT ACC } & PDD & $86(15)$ & $80(18)$ & $80(17)$ & 82 (17) & $W L=0.913$ & $F_{2,56}=25.411$ & $W L=0.965$ \\
\hline & DLB & $83(10)$ & $76(17)$ & $78(14)$ & $75(21)$ & $F_{3,54}=1.707$ & $\begin{array}{l}\mathrm{p}<0.001 \\
\mathrm{ES}=0.476\end{array}$ & $\begin{array}{l}F_{6,108}=0.325 \\
p=0.923\end{array}$ \\
\hline & PD & $96(5)$ & $96(4)$ & $96(4)$ & $97(3)$ & $\begin{array}{l}p=0.177 \\
E S=0.087\end{array}$ & & $\mathrm{ES}=0.018$ \\
\hline \multirow[t]{3}{*}{ CVAR CRT } & PDD & $0.40(0.20)$ & $0.59(0.44)$ & $0.45(0.27)$ & $0.43(0.22)$ & $W L=0.809$ & $F_{2,56}=14.465$, & $W L=0.815$ \\
\hline & DLB & $0.44(0.20)$ & $0.49(0.19)$ & $0.394(0.192)$ & $0.49(0.21)$ & $\mathrm{F}_{3,54}=4.243$ & $\begin{array}{l}p<0.001 \\
E S=0.341\end{array}$ & $\begin{array}{l}F_{6,108}=1.944 \\
p=0.080\end{array}$ \\
\hline & PD & $0.21(0.05)$ & $0.23(0.08)$ & $0.22(0.08)$ & $0.25(0.011)$ & $\begin{array}{l}p=0.009 \\
E S=0.191\end{array}$ & & $\mathrm{ES}=0.097$ \\
\hline \multirow[t]{3}{*}{ COGRT } & PDD & $522(564)$ & $822(1189)$ & 706 (1115) & 650 (1052) & $\mathrm{WL}=0.912$ & $F_{2,47}=9.934$ & $W L=0.820$ \\
\hline & DLB & $362(982)$ & $-156(866)$ & 603 (1761) & 572 (1079) & $F_{3,45}=1.442$ & $\begin{array}{l}\mathrm{p}<0.001 \\
\mathrm{ES}=0.297\end{array}$ & $\begin{array}{l}F_{6,90}=1.563 \\
p=0.167\end{array}$ \\
\hline & PD & 201 (99) & $202(78)$ & $168(58)$ & $151(44)$ & $\begin{array}{l}p=0.243 \\
E S=0.088\end{array}$ & & $\mathrm{ES}=0.094$ \\
\hline \multirow[t]{3}{*}{ RDS } & PDD & $3.9(1.8)$ & $3.6(2.0)$ & $4.1(2.2)$ & $4.1(2.1)$ & $\mathrm{WL}=0.917$ & $F_{2,57}=16.20$ & $W L=0.901$ \\
\hline & DLB & $3.6(1.6)$ & $4.0(1.2)$ & $3.5(1.7)$ & 3.9 (1.3) & $F_{3,55}=1.65$ & $\begin{array}{l}p<0.001 \\
E S=0.362\end{array}$ & $F_{6,110}=0.985$ \\
\hline & PD & $6.0(1.5)$ & $6.6(2.0)$ & $6.6(2.2)$ & $6.7(2.1)$ & $\begin{array}{l}p=0.188 \\
E S=0.083\end{array}$ & & $\begin{array}{l}p=0.439 \\
E S=0.051\end{array}$ \\
\hline \multirow[t]{3}{*}{ VAS } & PDD & 45 (16) & $55(21)$ & $52(20)$ & $58(23)$ & $W L=0.786$ & $F_{(2,55)}=6.83$ & $W L=0.861$ \\
\hline & DLB & $55(21)$ & $55(21)$ & $57(23)$ & $65(23)$ & $\mathrm{F}_{3,53}=4.804$ & $\begin{array}{l}p=0.002 \\
E S=0.199\end{array}$ & $F_{6,106}=1.370$ \\
\hline & PD & $68(17)$ & $66(19)$ & 72 (13) & 75 (14) & $\begin{array}{l}p=0.005 \\
E S=0.214\end{array}$ & & $\begin{array}{l}p=0.233 \\
E S=0.072\end{array}$ \\
\hline \multirow[t]{3}{*}{ UPDRS III } & PDD & 43 (12) & $36(8)$ & $35(10)$ & $38(10)$ & $W L=0.494$ & $F_{2,57}=3.44$ & $W L=0.839$ \\
\hline & DLB & $36(10)$ & $33(10)$ & $33(9)$ & $34(10)$ & $F_{3,55}=18.8$ & $\begin{array}{l}p=0.039 \\
E S=0.108\end{array}$ & $F_{6,110}=1.680$ \\
\hline & PD & $36(9)$ & $29(9)$ & $30(9)$ & $30(8)$ & $\begin{array}{l}\mathrm{p}<0.001 \\
\mathrm{ES}=0.506\end{array}$ & & $\begin{array}{l}p=0.133 \\
E S=0.084\end{array}$ \\
\hline
\end{tabular}

ChEls, cholinesterase inhibitors; cognition reaction time CRT, choice reaction time; CRT ACC, CRT response accuracy; CRT SD, cognition reaction time standard deviation; CVAR CRT, coefficient of variation in choice reaction time; DLB, dementia with Lewy bodies; DPIC RT, delayed picture recognition reaction time; DVIG RT, digit vigilance reaction time; ES, eta squared; MMSE, Mini-Mental State Examination; NPI, Neuropsychiatric Inventory; NWM RT, numeric working memory reaction time; PD, Parkinson's disease; PDD, Parkinson's disease with dementia; RDS, reverse digit span; SRT, simple reaction time; UPDRS, Unified Parkinson's Disease Rating Scale; VAS, Visual Analogue Scale; WL,Wilk's.

*Within-subject effects examine the effect of treatment with time (expressed as WL, $F_{d f}$, df error, $p, E S$ ).

†Between-subject effects examine the differences between diagnostic groups (expressed as $F_{d f}, d f$ error, $p, E S$ ).

fInteraction effects examine the combination of diagnosis with time (expressed as WL, $F_{d f, d f}$ error, $p, E S$ ).

SRT, CRT, CRTSD, COG RT, measured in ms. CRT ACC expressed as a percentage.

acute L-dopa challenge, most cognitive measures did not change markedly. Subjective alertness and UPDRS III scores improved to a greater degree in patients with Parkinson's disease than in those with PDD. Over the $2 \mathrm{~h}$ after treatment with L-dopa a marked change in level of fluctuating attention was detected, but this was not in any particular direction and most likely reflects fluctuation in CRT performance despite controlling for the mean performance levels in this task. This is a recognised feature in patients with both PDD and DLB, and this study has replicated previous work by suggesting that both conditions are indistinguishable in CRT, CRT SD or COG RT, but are considerably different in patients with Parkinson's disease. ${ }^{4}$ An interaction between time point and diagnosis for measures of within-trial variability (CRT and CRT SD) could simply represent increased fluctuation in attention in patients with dementia compared with patients with Parkinson's disease. The alternative explanation, that
L-dopa caused increased fluctuation in patients with dementia, is, however, impossible to exclude. As with the acute data, most cognitive measures did not change considerably with L-dopa use over 3 months although mean SRT and DVIG RT worsened in patients with PDD, whereas mean RDS scores deteriorated in the Parkinson's disease group (despite an apparent improvement in mean MMSE). However, with sensitivity analysis showing no marked individual patient deterioration in this time, these results are of uncertain significance. Overall, neuropsychiatric function improved in both patients with Parkinson's disease and PDD, but showed no change in those with DLB, as shown by NPI scores. It is, however, conceivable that an improvement in some scores was masked by concomitant deterioration in others on this measure.

Striatal dopaminergic function reduces by $6-10 \%$ per decade from early to late adulthood. ${ }^{25}$ Cognitive decline in patients with Parkinson's disease may be influenced by the 
degree of motor impairment and hence the response to Ldopa, whereas motor response may decrease with the development of dementia in patients with Parkinson's disease. ${ }^{26}$ We did not find any difference in motor response to acute L-dopa challenge between Parkinson's disease and PDD groups although the PDD group were younger and received a higher mean dose of L-dopa. Alternatively, dementia may impair UPDRS III performance and the ability to perform manually based cognitive tasks, and severe cognitive impairment may have an alternative dopamine response. Previous studies have suggested a link between bradyphrenia in Parkinson's disease and simultaneous cognitive task performance, as shown by slower CRT tasks. ${ }^{27}$ The level of cognitive slowing may correspond to the level of independently assessed motor slowing, raising the possibility that cognitive impairment may reflect dysfunction in the striatum or premotor cortex. ${ }^{28}$

The most profound short-term and long-term cognitive deficit in animals with MPTP (1-methyl-4-phenyl-1,2,5,6tetrahydropyridine)-induced parkinsonism is impaired spatial working memory, representing damage in the frontostriatal system. ${ }^{29}$ L-dopa administration to MPTP-treated monkeys can considerably ameliorate these impairments. ${ }^{30}$ Similarly, dopamine withdrawal in patients with Parkinson's disease can highlight selective frontal lobe dysfunction, particularly spatial working memory, executive function, and thinking time and accuracy. ${ }^{31}$ L-dopa replacement in patients with Parkinson's disease improves aspects of working memory, particularly visuospatial and object tasks, but by contrast, apomorphine can worsen reaction times without affecting the accuracy. ${ }^{32}$ These results may represent preferential dopaminergic receptor activation and are supported by animal studies, which suggest that Dl dopamine receptor agonist infusion enhances attention in rats, with similar results reported for L-dopa and D2 receptor antagonists in humans. ${ }^{33}$ In this study, neither visuospatial tasks (represented by picture recognition) nor numeric working memory changed after 3 months of L-dopa treatment.

Executive function includes the inhibition of inappropriate responses to external stimuli. Increased dopamine levels have been linked to an increased frequency of premature response and thus decreased accuracy of response by diminishing the ability to suppress the wrong response, reflecting impulsivity. ${ }^{34}$ Other studies have, however, failed to detect either change in reaction time responses or working memory subsequent to dopamine administration. ${ }^{35}{ }^{36}$ Furthermore, CRTs have been reported by others to deteriorate in patients with Parkinson's disease after acute L-dopa challenge, potentially due to a sedative effect of treatment. ${ }^{37}$ Therefore, the acute effect of L-dopa administration on reaction time and accuracy in dopamine-depleted conditions remains unclear. We found no adverse acute effect of L-dopa on any aspect of cognitive function in our Parkinson's disease cohort, including reaction times and accuracy. However, the mean acutely administered dose of L-dopa was less than that recommended in acute motor challenges, possibly accounting for the lack of change in reaction times and also the maintenance of CRT accuracy.

Apparently conflicting data on the role of dopamine on cognition may be reconciled through animal studies which show that insufficient as well as excessive dopaminergic stimulation in the prefrontal cortex impairs working memory. ${ }^{8}$ Baseline cognitive performance can influence the effect of dopaminergic drugs. Hence, in patients with Parkinson's disease, treatment with L-dopa can have both beneficial and deleterious effects on cognitive function depending on the task assessed and the underlying basal corticostriatal dopaminergic function. ${ }^{38}$ Although no adverse acute effect of L-dopa on cognition was found in the present study, the results were limited by submaximal acute L-dopa dosing. Future studies may replicate this "inverted U" dose-related cognitive response curve for patients with parkinsonism and dementia.

Although objective measures remained unchanged in our study, subjectively patients with DLB and PDD felt more alert, despite increased fluctuating cognition. Reduced SRT or CRT did not accompany this acute improvement in subjective alertness in any group. The beneficial effect of L-dopa on sense of alertness could reflect concomitant motor benefit, but against this is the fact that these values did not temporally correlate. A dissociable motor and cognitive effect on withdrawal of dopaminergic drugs has been suggested previously, ${ }^{39}$ with support from functional imaging data which has shown that dopamine modulates cognitive and motor function by separate pathways, with direct dopaminergic input to the prefrontal cortex facilitating working memory via the mesocortical circuits. ${ }^{40}$

Our study has several methodological flaws, including an open-label design, small sample size, use of modest L-dopa dosing in acute challenges, particularly to patients with DLB, and the recognised inadequacies of an overnight fast in excluding the long duration response to L-dopa. Also, most patients with dementia were receiving ChEIs, and the study was therefore unable to deal with the effect of L-dopa on cognition in parkinsonism with dementia in ChEI-naive patients, or the potential interactions between ChEIs and Ldopa. We conclude that L-dopa does not have any clinically significant adverse cognitive or behavioural effects in patients with PDD. Furthermore, the cautious use of L-dopa in DLB is not contraindicated when increasing severity of motor impairment warrants treatment.

\section{Authors' affiliations}

S A Molloy, E N Rowan, J T O'Brien, I G McKeith, D J Burn, Institute of Ageing and Health, Wolfson Research Centre, Newcastle General Hospital, Newcastle upon Tyne, UK

K Wesnes, Cognitive Drug Research (CDR), Goring on Thames, UK

Competing interests: None declared.

\section{REFERENCES}

1 Aarsland D, Andersen K, Larsen JP, et al. Risk of dementia in Parkinson's disease: a community based, prospective study. Neurology 2001;56:730-6.

2 Papka M, Rubio A, Schiffer RB. A review of Lewy body disease, an emerging concept of cortical dementia. J Neuropsychiatr Clin Neurosci 1998;10:267-79.

3 Mahieux F, Fenelon G, Flahault A, et al. Neuropsychological prediction of dementia in Parkinson's disease. J Neurol Neurosurg Psychiatry 1998:64:178-83.

4 Ballard CG, Aarsland D, McKeith IG, et al. Fluctuations in attention: PD dementia vs DLB with parkinsonism. Neurology 2002;59:1714-20.

5 Aarsland D, Ballard C, Larsen JP, et al. A comparative study of psychiatric symptoms in dementia with Lewy bodies and Parkinson's disease with and without dementia. Int J Geriatr Psychiatry 2001;16:528-36.

6 Mc Keith IG, Galasko D, Kosaka K, et al. Consensus guidelines for the clinical and pathologic diagnosis of dementia with Lewy bodies (DLB): report on the consortium on DLB international workshop. Neurology 1996:47:1113-24.

7 Aarsland D, Larsen JP, Lim NG, et al. Range of neuropsychiatric disturbances in patients with Parkinson's disease. J Neurol Neurosurg Psychiatry 1999;67:492-6.

8 Cools R, Barker RA, Sahakian BJ, et al. L-dopa medication remediates cognitive inflexibility, but increases impulsivity in patients with Parkinson's disease. Neuropsychologia 2003;41:1431-41.

9 Molloy S, McKeith IG, O'Brien JT, et al. The role of levodopa in the management of dementia with Lewy bodies. J Neurol Neurosurg Psychiatry 2005;76:1200-3.

10 McKeith IG, Del Ser T, Spano P, et al. Efficacy of rivastigmine in dementia with Lewy bodies; a randomised, double blind, placebo controlled, international study. Lancet 2000;356:2031-6.

11 Emre M, Aarsland D, Albanese A, et al. Rivastigmine for dementia associated with Parkinson's disease. N Engl J Med 2004;351:2509-18.

12 Okereke CS, Kirby L, Kumar D, et al. Concurrent administration of donepezil $\mathrm{HCl}$ and levodopa/carbidopa in patients with Parkinson's disease: assessment of pharmacokinetic changes and safety following multiple oral doses. $\mathrm{Br} J \mathrm{Clin}$ Pharmacol 2004;58:41-9.

13 Gibb WRG, Lees AJ. The relevance of the Lewy body to the pathogenesis of idiopathic Parkinson's disease. J Neurol Neurosurg Psychiatry 1988;51:745-52. 
14 American Psychiatric Association (APA). Diagnostic and statistical manual of mental disorders, fourth edition (text revision; DSM-IV-TR). Washington, DC: American Psychiatric Association, 2000.

15 Folstein M, Folstein S, McHugh PR. "Mini-mental state". A practical method for grading the cognitive state of patients for the clinician. J Psychiatr Res $1975 ; 12: 189-98$

16 Cummings JL, Mega M, Grey K, et al. The Neuropsychiatry Inventory: comprehensive assessment of psychopathology in dementia. Neurology 1994:44:2308-14.

17 Bond $\mathbf{A}$, Lader $M$. The use of analogue scales in rating subjective feelings. Br J Psychol 1974;47:211-18.

18 Weschler D. Weschler adult intelligence scale manual. New York: Psychological Corporation, 1955.

19 Simpson PM, Surmon DJ, Wesnes KA, et al. The Cognitive Drug Research computerised assessment system for demented patients: a validation study. Int J Geriatr Psychiatry 1991;6:95-102.

20 Walker MP, Ayre GA, Cummings JL. The clinician's assessment of fluctuations and the one day fluctuating assessment scale. Two methods to assess fluctuating confusion in dementia. Br J Psychiatry 2000;177:252-6.

21 Nutt JG. Response to L-dopa in PD: the long and the short of it. Neurology 2000;54:1884-5

22 Albanese A, Bonucelli U, Brefel C, et al. Consensus statement on the role of acute dopaminergic challenge in Parkinson's disease. Mov Disord 2001;16:197-201.

23 Fahn S, Elton RL and the members of the UPDRS development committee. Unified Parkinson's Disease Rating Scale. In: Fahn S, Marsden CD, Calne DB, eds. Recent developments in Parkinson's disease. London: Macmillan, 1987:153-63.

24 Clarke CE, Davies P. Systematic review of acute levodopa and apomorphine challenge tests in the diagnosis of idiopathic Parkinson's disease. J Neurol Neurosurg Psychiatry 2000;69:590-4.

25 Backman L, Ginovart N, Dixon RA, et al. Age related cognitive deficits mediated by changes in the striatal dopamine system. Am J Psychiatry 2000;157:635-7.

26 Apayadin H, Ahlskog JE, Parisi JE, et al. Parkinson disease neuropathology: later developing dementia and loss of the levodopa response. Arch Neurol 2002;59:102-12
27 Malapani C, Pillon B, Dubois B, et al. Impaired simultaneous cognitive task performance in Parkinson's disease: a dopamine related dysfunction. Neurology 1994;44:319-25.

28 Sawamoto N, Honda M, Hanakawa T, et al. Cognitive slowing in Parkinson's disease: a behavioural evaluation independent of motor slowing. J Neurosci 2002;22:5198-203.

29 Fernandez-Ruiz J, Doudet DJ, Aigner TG. Long-term cognitive impairment in MPTP-treated rhesus monkeys. Neuroreport 1995;29:102-4.

30 Fernandez-Ruiz J, Doudet DJ, Aigner TG. Spatial memory improvement by levodopa in parkinsonian MPTP-treated monkeys. Psychopharmacology (Berl) 1999; 147:104-7.

31 Lange KW, Robbins TW, Marsden CD, et al. L-dopa withdrawal in Parkinson's disease selectively impairs cognitive performance in tests sensitive to frontal lobe dysfunction. Psychopharmacology (Berl) 1992;107:394-404.

32 Costa A, Peppe A, Dell'Agnello G, et al. Dopaminergic modulation of visualspatial working memory in Parkinson's disease. Dement Geriatr Cogn Disord 2003; 15:55-66.

33 Robbins TW. Chemical neuromodulation of frontal-executive functions in humans and other animals. Exp Brain Res 2000;133:130-8.

34 Lecourtier L, Kelly PH. Bilateral lesions of the habenula induce attentional disturbances in rats. Neuropsychopharmacology 2005;30:484-96.

35 Jahanshahi M, Brown RG, Marsden CD. Slowness in motor readiness and motor programming may not be specific to striatal dopamine. J Neurol Neurosurg Psychiatry 1992;55:1 168-76.

36 Press DZ, Mechanic DJ, Tarsy D. Cognitive slowing in Parkinson's disease resolves after practice. I Neurol Neurosurg Psychiatry 2002;73:524-8.

37 Muller T, Benz S, Przuntek H. Choice reaction time after levodopa challenge in parkinsonian patients. J Neurol Sci 2000;181:98-103.

38 Arnsten AFT. Catecholamine modulation of prefrontal cortical cognitive function. Trends Cogn Sci 1998;2:436-46.

39 Fern-Pollack L, Whone AL, Brooks DJ, et al. Cognitive and motor effects of dopaminergic medication withdrawal in Parkinson's disease. Neuropsychologia 2004:42:1917-26.

40 Mattay VS, Tessitore A, Callicott JH, et al. Dopaminergic modulation of cortical function in patients with Parkinson's disease. Ann Neurol 2002;51:156-64.

\section{NEUROLOGICAL PICTURE}

\section{Extensive brain calcification in idiopathic hypoparathyroidism}

A 24-year-old-woman showed confusion, movement disorders and asthenia related to severe hypocalcaemia $(0.95 \mathrm{mmol} / \mathrm{l})$, with low parathyroid hormone concentration $(4 \mathrm{pg} / \mathrm{ml}$, normal $15-$ $85 \mathrm{pg} / \mathrm{ml}$ and hyperphosphataemia $(1.97 \mathrm{mmol} / \mathrm{l})$. She was hospitalised for depression 1 year previously, but no biological examination was performed during that period. Six years previously, asymptomatic hypocalcaemia ( $1.48 \mathrm{mmol} / \mathrm{l})$ was diagnosed and the computed tomography scan at that time was normal. Regarding biological explorations, the final diagnosis was idiopathic hypoparathyroidism. Calcium and vitamin replacement were prescribed at that time, but the patient did not follow it up.

The mechanism of intracranial calcification in hypoparathyroidism, ${ }^{1}$ more often seen in pseudohypoparathyroidism than in idiopathic hypoparathyroidism, has not been completely elucidated. It may be related more to the duration of hypocalcaemia and hyperphosphataemia than parathyroid hormone itself. Hyperphosphataemia promotes ectopic calcification in brain tissue in hypoparathyroidism.

A non-enhanced computed tomography scan shows bilateral and symmetrical calcifications in basal ganglia (mainly globus pallidus), cerebellum (dentate nuclei) and at the grey-white junction (fig 1). The extent of calcification is variable, depending on the stage of the disease, duration of metabolic abnormalities and volume of calcium deposit.

A computed tomography scan allows earlier diagnosis, with high sensitivity and specificity. Magnetic resonance imaging is not useful, as the signal intensity of calcified lesions varies widely.

Clinical symptoms of hypoparathyroidism include tetany, painful muscle spasm of hands and feet, facial muscle spasms,

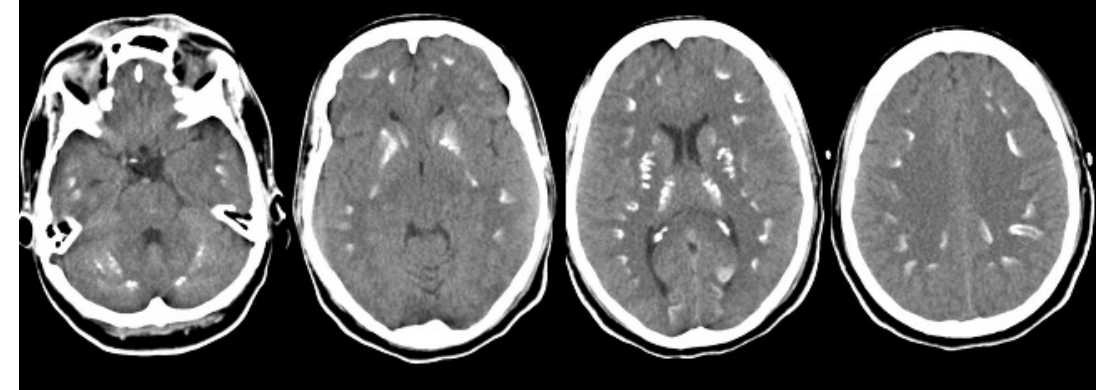

Figure 1 Non-contrast brain computed tomography scan showing extensive and symmetrical calcifications located at the grey-white junction and in the globus pallidi.

seizure. Hypoparathyroidism is also often associated with psychiatric symptoms, mainly delirium, but also cognitive impairment, ${ }^{2}$ psychosis, depression or anxiety.

Other diagnoses ${ }^{3}$ that should be evoked on a computed tomography scan include:

- Fahr disease

- basal ganglia calcifications in the case of trisomy 21 or 5, Cockayne syndrome, radiation therapy or intrathecal chemotherapy

- hyperparathyroidism

- neurolupus

M Mejdoubi, T Zegermann

Department of Neuroradiology, Purpan Hospital, Toulouse, France

Correspondence to: Dr M Mejdoubi, Department of Neuroradiology, Hôpital de Purpan, Place du Dr Baylac, 31059 Toulouse Cedex 9, France; mejdoubi.m@chu-toulouse.fr

Competing interests: None declared.

\section{REFERENCES}

1 Fujita T. Mechanism of intracerebral calcification in hypoparathyroidism. Clin Calcium 2004;14:55-7.

2 Kowdley KV, Coull BM, Orwoll ES. Cognitive impairment and intracranial calcification in chronic hypoparathyroidism. Am J Med Sci 1999:317:273-7.

3 Ogi S, Fukumitsu N, Tsuchida D, et al. Imaging of bilateral striopallidodentate calcinosis. Clin Nucl Med 2002;27:721-4 